Archives de sciences sociales des religions

156 | octobre-décembre 2011

Bulletin Bibliographique

\title{
Philippe Béguerie, Vers Écône. Mgr Lefebvre et les Pères du Saint-Esprit
}

\section{Paul Airiau}

\section{(2) OpenEdition}

Journals

Édition électronique

URL : http://journals.openedition.org/assr/22866

DOI : $10.4000 /$ assr.22866

ISSN : 1777-5825

Éditeur

Éditions de l'EHESS

Édition imprimée

Date de publication : 31 décembre 2011

Pagination : 106

ISBN : 9782713223273

ISSN : 0335-5985

\section{Référence électronique}

Paul Airiau, «Philippe Béguerie, Vers Écône. Mgr Lefebvre et les Pères du Saint-Esprit », Archives de sciences sociales des religions [En ligne], 156 | octobre-décembre 2011, document 156-15, mis en ligne le 14 février 2012, consulté le 21 septembre 2020. URL : http://journals.openedition.org/assr/22866 ; DOI : https://doi.org/10.4000/assr.22866

Ce document a été généré automatiquement le 21 septembre 2020.

(c) Archives de sciences sociales des religions 


\title{
Philippe Béguerie, Vers Écône. Mgr Lefebvre et les Pères du Saint-Esprit
}

\author{
Paul Airiau
}

\section{RÉFÉRENCE}

Philippe BÉGUERIE, Vers Écône. Mgr Lefebvre et les Pères du Saint-Esprit, Postface de Florian Michel. Paris, Desclée de Brouwer, coll. « Pages d'Histoire-Document », 2010, $479 \mathrm{p}$.

1 Avant d'être l'évêque rebelle que l'on sait, Mgr Marcel Lefebvre fut Père du Saint-Esprit et supérieur de sa congrégation, de 1962 à 1968 . Son passage suscita des ruptures parmi ses confrères. Le P. Philippe Béguerie quitta ainsi les spiritains, en 1963, pour devenir un spécialiste de liturgie.

2 Il propose ici un ouvrage qui présente plusieurs intérêts. Pour la forme d'abord: il associe récit historique et publication de pièces d'archives et de documents, qui permettent de mieux comprendre Lefebvre et les spiritains dans les années soixante et que l'évêque lefebvriste Bernard Tissier de Mallerais, dans sa bio-hagiographie Marcel Lefebvre. Une vie (Clovis, 2002), n'avait pu consulter, les archives spiritaines ne lui ayant pas été ouvertes. Il faut remercier Desclée de Brouwer d'avoir pris le risque d'éditer ce recueil de documents, malgré le faible espoir de retour financier de toute opération de ce type. Sont ainsi mises à disposition des pièces spiritaines dont il faut connaître ou soupçonner l'existence pour les utiliser et, trop peu de chercheurs exploitant les riches fonds spiritains, il faut aussi remercier les Archives générales de la Congrégation du Saint Esprit d'en avoir accepté la publi-cation.

3 Pour l'angle choisi ensuite. P. Béguerie propose un témoignage qui est en même temps un récit historique: une articulation de ses souvenirs et des documents d'archives (donnés dans un ordre qui n'est pas toujours chronologique), au service d'une œuvre de vérité - dans les deux sens du terme : écrire, selon les canons de l'histoire universitaire, 
ce qui fut, et justifier - non excuser, mais montrer que ce fut juste, d'un point de vue ecclésiologique et théologique - son opposition à Lefebvre.

4 Je relèverai ici que la partie (oh combien difficile !) n'est pas complètement gagnée. Ainsi, les documents ne prouvent pas toujours ce qui est avancé. Prenons deux points en relation qui importent pour l'interprétation que l'on donne du parcours de Lefebvre. D'abord, l'opposition de Jean XXIII au prélat, rapportée par un spiritain (à quelle date ?) Elle remonterait au sacre de Lefebvre, en 1947, car il avait loué le P. Henri Le Floch, son ancien supérieur du séminaire français de Rome, renvoyé pour sa résistance à la condamnation de l'Action française, (p. 56-57, 167-172). L'affirmation aurait pu être vérifiée dans les carnets d'Angelo Roncalli édités par Étienne Fouilloux (Journal de France I. 1945-1948, Paris, Éditions du Cerf, 2006, p. 411, 558, 560). Or, le nonce était en vacances le jour du sacre. Il reçoit Lefebvre un an plus tard, les 15 et 19 octobre 1948, sans mentionner le contenu de leur échange. Fouilloux, en se fondant sur Tissier de Mallerais, considère l'hostilité comme avérée et se demande si le reproche aurait été formulé le 15 octobre. Il n'y a donc pas de trace écrite, seulement une tradition spiritaine et une tradition lefebvriste (qui ont peut-être la même origine). Leur convergence pourrait plaider en la faveur de l'authenticité, mais cela peut aussi provenir d'une relecture a posteriori liée à l'itinéraire de Lefebvre, pour en montrer la cohérence, en bien ou en mal selon les opinions. Mais faut-il vraiment postuler une cohérence?

5 Second point lié à cette linéarité évidente, trop évidente, et renvoyant à une interprétation politique du lefebvrisme: Le Floch, et on excusera une intrusion fort personnelle. Ainsi qu'il ressort de mes recherches (Le Séminaire français de Rome $d u$ P. Le Floch, 1904-1927, thèse d'histoire, IEP de Paris, 2003), ce spiritain n'a jamais conservé par-devers lui le dossier de condamnation de l'Action française (par l'Index et non le Saint Office; en 1914 et non en 1926, p. 58-59). Il en fut accusé par l'évêque d'Oran, Mgr Durand, sans aucun fondement, et l'assertion se propagea jusqu'à aujourd'hui.

6 Mettre en avant cette influence politique et religieuse, c'est, je le répète, postuler une continuité qui n'est pas évidente - interpréter Lefebvre en fonction de ce qu'il est devenu, et non en fonction de ce qu'il a été successivement. Après tout, pourquoi Gabriel Garrone - qui joua un rôle dans la rupture Rome-Lefebvre - n'a-t-il pas suivi les positions de Lefebvre alors qu'ils furent condisciples au Sémi-naire français ? Pourquoi a-t-il été influencé par Jacques Chevalier alors qu'au séminaire français il adhérait à l'esprit intransigeant de Le Floch ? Pourquoi Alfred Ancel n'a-t-il jamais été critiqué par Jean XXIII, alors qu'il avait, lui aussi, publiquement reconnu ce qu'il devait à Le Floch ? Et pourquoi de tous les prêtres passé à Rome sous Le Floch, fort peu se rallièrent à l'intégrisme? Il est beau de poser une linéarité, encore faudrait-il la démontrer réellement, par delà les affirmations des acteurs, et en même temps rendre compte du changement de ceux qui ont changé. Car, après tout, c'est peut-être Lefebvre qui a changé, en refusant le changement de Vatican II, alors que ses confrères changeaient pour ne pas changer - pour rester romains, de la romanité imposée par Pie XI : suivre le pape même lorsqu'il paraît modifier les positions du magistère antérieur.

7 Je regretterai enfin que ce récit-étude n'ait pas pu, pour des raisons indépendantes de la volonté de l'auteur, être confronté à l'analyse faite par Luc Perrin des élections du supérieur général spiritain en 1962 et 1968 ("Mgr Lefebvre, d'une élection à une démission (1962-1968)», Histoire et missions chrétiennes, "Action française. 
Décolonisation. Mgr Lefebvre », 10, 2009, p. 139-172). Tous deux avancent qu'une action pro-lefebvriste a été menée par des membres de l'administration supérieure de la congégration, et s'accordent sur l'enjeu de l'élection de 1962 : remettre au pas le noviciat de Chevilly, jugé trop avancé théologiquement et pastoralement - noviciat où était affecté P. Béguerie.

Bref, ces quelques éléments très ponctuels amènent à nuancer en partie l'affirmation finale de Florian Michel, dans la postface, sur « la force du dialogue entre un acteur et des documents d'archive ». Si le travail archivistique est important, il n'est pas achevé - ou plutôt, l'exploitation historienne est à reprendre et à poursuivre. L'apport à la préhistoire du lefebvrisme est pourtant important, et il ne faut pas gâcher son plaisir, ne serait-ce que parce que l'auteur permet de mieux appréhender le supériorat général de Mgr Lefebvre, et donne à réfléchir sur son parcours, alors que se déroule Vatican II.

Cette postface de Florian Michel appelle à une véritable histoire de l'intégrisme catholique français. Comment ne pas souscrire à un tel vœu, puisque, et j'accentuerai ici la dimension personnelle de cette recension, je porte le même depuis mon entrée dans la carrière universitaire, il y a plus de quinze ans? F. Michel me permettra de discuter ici rapidement certaines de ses idées, pour poursuivre un débat déjà entamé en privé.

10 Cinq points. D'abord, la question politique, un des plus gros morceaux. Pour lui, la racine politique de l'affaire lefebvriste ne peut être occultée : le maurrassisme est une matrice de l'intégrisme. La thèse est ancienne, développée par Louis Canet sous le pseudonyme de Nicolas Fontaine (Saint Siège, Action française et "catholiques intégraux", Paris, Librairie Universitaire J. Gamber, 1928), théologisée et historicisée par le P. Yves Congar op, en 1950 («Mentalité de droite et intégrisme », La Vie intellectuelle), puis par René Rémond ("Droite et gauche dans le catholicisme français contemporain », Revue Française de Science Politique, VIII-3, 1958, p. 529-544 ; VIII-4, 1958, p. 803-820). F. Michel l'enrichit en passant du contenu politique aux méthodes politiques : l'intégrisme utilise des techniques d'action venues de la politique. Cependant, le raisonnement peut être inversé : l'intégrisme n'est pas la traduction théologique de positions politiques mais le philo-maurrassisme ou l'appui à l'extrême-droite est la traduction politique de positions théologiques - car, pour Lefebvre, fort orthodoxe sur ce point, le politique est au service de la conformation religieuse de la société, et l'on peut donc en utiliser les méthodes (d'où l'appui à la Cité Catholique qui jouera beaucoup dans la distance creusée entre évêques français, certains spiritains et Lefebvre). De tous les cas de ténors ou mentors intégristes que j'ai pu étudier, seul l'abbé Georges de Nantes relèverait d'un intégrisme issu du maurrassisme. Ailleurs, j'ai observé les racines religieuses de positions politiques, et même l'abandon de racines politiques aux positions religieuses pour passer à un enracinement théologique du pro-maurrassisme (ainsi l'abbé Victor-Alain Berto, théologien de Lefebvre à Vatican II). Qu'en est-il des «troupes» intégristes? Le recrutement premier, dans les années soixante-soixantedix, provient-il d'abord de milieux culturellement maurrassiens, ou bien l'acculturation à ce qui devient l'intégrisme conduit-elle à une intégration d'un philo-maurrassisme l'intégrisme continuant à considérer que la condamnation fut une erreur qui cassa les chances de rétablir une chrétienté en France ? La question reste à étudier.

11 Puis je ne rejetterai pas si immédiatement l'interprétation proposée par Luc Perrin sur «l'aggiornamento de type pacellien » voulu par Lefebvre, même si la formule est volontairement une formule. Lorsque ce dernier écrit dans sa lettre aux spiritains du 
11 octobre 1962, à l'occasion de l'ouverture de Vatican II : « Que l'Esprit Saint vivant en nous nous fasse prendre une conscience toujours plus vive de notre appartenance à l'Église tout entière encore soumise au souffle et au feu de la Pentecôte (...) Aujourd'hui encore cette Pentecôte continue et va apparaître d'une manière plus sensible à l'occasion du Concile» (p.279), il est difficile de ne pas y voir une thématique roncallienne renouvelant Pie XII finissant, et la participation à un courant plus large entamé à la fin du XIX ${ }^{\mathrm{e}}$ siècle, présent du Mexique à la France, de Conception Cabrera de Arminda à Marthe Robin, en passant par Léon XIII et l'Italienne Elena Guerra. Certes, la suite de la lettre articule cette thématique du renouveau spirituel par l'Esprit à des habitus ecclésiastique fort classiques. Mais Jean XXIII et Pie XII faisaient-ils autre chose?

Ensuite, pour intégrer Lefebvre dans l'histoire de l'intégrisme, il ne faut jamais oublier qu'il n'est pas, entre 1962 et 1968, le ténor de l'intransigeantisme français à tendance apocalyptique. Au niveau ecclésiastique, les abbés Georges de Nantes, Jean Boyer, Louis Coache, le P. Noël Barbara, sont plus célèbres que lui et attirent largement l'attention médiatique - sans oublier les laïcs fort offensifs, tels Louis Jugnet, et les revues Itinéraires de Jean Madiran ou La Pensée catholique de l'abbé Luc J. Lefèvre. Il y aura un jour à expliquer comment et quand Lefebvre a pu et su, après Vatican II, prendre l'ascendant dans le milieu devenu intégriste - ne serait-ce que parce qu'il fut en mesure de fournir des prêtres adéquats à ceux qui les demandaient. Il y aura aussi à expliquer pourquoi la Cité Catholique renouvelée au milieu des années soixante ne se prononce pas sur les questions issues du Concile. La principale organisation de l'intégrisme cède le champ "médiatique " à des organismes qui vont se positionner au plan religieux d'abord et avant tout. Et la frontière entre ce qui est intégrisme et ce qui ne l'est pas est alors fort floue : où situer Credo, les Silencieux de l'Église, Fidélité et Ouverture, Pierre Debray, Michel de Saint Pierre et alii?

Encore, cette histoire est ancienne. Elle ne peut débuter en 1962 ou en 1968, ni même en 1945. La matrice est l'intransigeance du début du Xx ${ }^{\mathrm{e}}$ siècle, avec la crise moderniste. La condamnation de l'Action française tranche dans le vif entre diverses options dans le catholicisme français. La structuration d'un certain nombre d'initiatives intransigeantes se déroule dans les années 1942-1946, et participe ainsi à ce que l'on appelle usuellement le renouveau catholique d'après-guerre. Et j'insisterai très fortement sur les effets religieux de la Guerre d'Algérie, revivifiant les lectures apocalyptiques alors qu'elles s'étaient atténuées dans les milieux intransigeants depuis une vingtaine d'années. F. Michel me rejoindra sur ce point, sans aucun doute: l'histoire de l'intégrisme doit débuter à la fin du XIX ${ }^{\mathrm{e}}$ siècle.

Sur ce dernier point, on relèvera que l'apocalyptisme joue sans doute un rôle-clé chez Lefebvre. Sa lettre sur les indépendances africaines, donnée au journal canadien Le Devoir et reprise dans La France catholique, le 18 décembre 1959, présente cette dimension : "il faut choisir pour le message de Fatima ou pour l'enfer d'ici-bas (...) il faudra bien un jour que [les hommes d'État] choisissent entre l'Étole et la Croix, entre l'enfer terrestre ou le paradis préparé ici-bas » (p. 74). La thématique reflue au milieu des années soixante, puis resurgit dans son article donné à Rivarol, le 7 mars 1968, qui traumatise nombre de spiritains français et fait grand bruit dans le monde médiatique. L'Église ayant résisté aux hérésies et erreurs lors de Vatican II, cela "déplaît souverainement non seulement aux ennemis traditionnels de l'Église inspiré par le Prince de ce monde (...). (...) quand on sait qu'au regard de ceux qui cherchent à 
dominer le monde, les communistes et les technocrates de la finance internationale, le seul véritable obstacle à l'asservissement de l'Église catholique est l'Église catholique et romaine, on ne sera pas surpris des efforts conjugués des communistes et des francsmaçons pour modifier et le magistère et la structure hiérarchique de l'Église » (p. 395). Satanisme, complotisme, antimaçonnisme, antilibéralisme, discret antijudaïsme : rien de neuf si ce n'est l'exploitation de ce registre, en 1968, par un évêque catholique alors que ces thèmes refluent depuis une quarantaine d'années. Enfin, dans la lettre de 1987 où il demandait à quatre de ses prêtres de se tenir prêts à recevoir le sacre épiscopal, il parlait explicitement des antéchrists de Rome. Bref, on retrouve la question : et si c'était Lefebvre qui avait changé, en restant apocalyptique alors que la thématique a reflué dès les années trente puis s'est effondrée avec Vatican II ? F. Michel avance en partie la chose, lorsqu'il replace l'évêque dans son contexte, celui d'une contestation généralisée, de la fondation charismatique de communautés nouvelles. Bref, une piste à creuser.

J'arrête ici cette longue recension: qu'on ne trouve d'autre explication à cette extension incongrue que l'intérêt de la lecture et le souci de participer à un débat. 